\title{
A Novel Musculoskeletal Joint Modeling for Orthopaedic Applications
}

\author{
Neriman Ozada ${ }^{1}$, Seyed Saeed Madani ${ }^{2}$ \\ ${ }^{1,2}$ Mechanical Engineering department, Eastern Mediterranean University, NORTH CYPRUS
}

\begin{abstract}
The objective of the work carried out in this research was to develop analytical and computational tools to model and investigate musculoskeletal human joints. It was recognised that the FEA was used by many researchers in modelling human musculoskeletal motion, loading and stresses. However the continuum mechanics played only a minor role in determining the articular joint motion, and its value was questionable. This is firstly due to the computational cost and secondly due to its impracticality for this application. On the other hand, there isn't any suitable software for precise articular joint motion analysis to deal with the local joint stresses or non standard joints. The main requirement in orthopaedics field is to develop a modeller software (and its associated theories) to model anatomic joint as it is, without any simplification with respect to joint surface morphology and material roperties of surrounding tissues. So that the proposed modeller can be used for evaluating and diagnosing different joint abnormalities but furthermore form the basis for performing implant insertion and analysis of the artificial joints. The work which is presented in this research is a new frame work and has been developed for human anatomic joint analysis which describes the joint in terms of its surface geometry and surrounding musculoskeletal tissues. In achieving such a framework several contributions were made to the $6 \mathrm{DOF}$ linear and nonlinear joint modelling, the mathematical definition of joint stiffness, tissue path finding and wrapping and the contact with collision analysis. In 6DOF linear joint modelling, the contribution is the development of joint stiffness and damping matrices. This modelling approach is suitable for the linear range of tissue stiffness and damping properties. This is the first of its kind and it gives a firm analytical basis for investigating joints with surrounding tissue and the cartilage. The 6DOF nonlinear joint modelling is a new scheme which is described for modelling the motion of multi bodies joined by non-linear stiffness and contact elements. The proposed method requires no matrix assembly for the stiffness and damping elements or mass elements. The novelty in the nonlinear modelling, relates to the overall algorithmic approach and handling local non-linearity by procedural means.
\end{abstract}

Key words: Musculoskeletal, Joint Modeling, Analytical and Computational Tools, Orthopaedic 


\section{INTRODUCTION}

Need for accurate musculoskeletal joint modeling It has been reported in the 5th Annual Report of National Joint Registry (NJR) on 31th March 2008 that in 2007/08, about 151,496, hip and knee joint replacement procedures were carried out in England and Wales in NHS and independent healthcare sector and about 583,724 total numbers of procedures were performed between 2003 and 2008. As the age of population in the western world gets older and as the life expectancy continues to rise, the need for joint replacement will also continue to increase. In addition to primary (first time a joint is replaced), the revision (repeated replacement) surgery will increase even further since increasing population with the replacement joint. The aging population is more prone to osteoarthritis, trauma, bone fracture and etc. Basically the prosthetic joint replacement is performed to recover the lost joint functionality. The joint replacement procedure has evolved since end of the 19th century. The most important factors involved in reconstructive surgery are the geometry of the prosthesis, type of prosthetic material and the positioning. In considering material types, since 1930s many material types such as stainless steel, titanium, vitalium, ceramic, platinum and polyethylene have been used in manufacturing of joint prosthesis (Pinchuk et al., 2005). The major problem with the prosthetic materials revealed with the accumulation of wear debries such as iron abscess. In addition to material based problems, replacement of a natural joint with an artificial prosthesis can kinematically alter the normal joint articulation due to modifying the natural centre of joint movements and forcing the joint to articulate relative to a fixed centre of movement. This is seen as the approximation of anatomical joints as idealised kinematic joints. Moreover, results from joint replacement also depend on the performance and capability of the surgeon which has high influence in positioning of prosthesis. However many of these issues still remain unsolved and there is a need for a more reliable joint replacement, less dependent on surgeon, with respect to right selection and right positioning of prosthesis which could restore the normal joint functionality. Most of the alteration and problems in joint replacement is due to the lack of scientific perspective of joint biomechanics In computational joint modelling and joint biomechanics, kinematics, musculoskeletal tissue behaviour, articulate contact, friction, and lubrication are analysed. The most important objectives in analysing the joint biomechanics are to understand the normal, abnormal, traumatic and prosthetic joint mechanisms along with surrounding tissue and contact interactions. As mentioned above, artificial joint loosening is a frequently seen complication. For example in elbow joint reconstruction, due to using semi constraint or constraint hinge joint prosthesis yield high number of dissatisfaction (instability or muscle loss) and joint loosening due to altered joint. Another commong problem injoint replacement is the incompatibleness between the natural joint and the artificial joint in terms of material properties. During the last fifty years or more research has gone into understanding of bone and tissue, surrounding a joint and their mechanical behaviour and mechanical and biological compatability with the implant material. Starting from early days where implants has progressed becoming more and more biocompatible with less loosening or stress shielding. However, joint kinematics has attracted much less attention compared to the biomaterials research, probably because motion kinematics was seen less important. Having a less natural mobility seems to be less life threatening than having an implant in body which can lead to body's rejection, infection or even cancer. 
Nevertheless, it became obvious that wrong kinematics does exactly the same thing by increasing friction and resulting a high wear debris, spread of implant molecules into body fluid or blood and local infection and may even lead to cancer. Attempts of understanding human body motion kinematics, is not new and gait analysis has been performed using specialised equipment and many analysis techniques had been developed. Analyis of human motion based on the musculoskeletal parameters and its dynamical equations are relatively new and generally based on modelling paradigms of mechanism modellers. Human body motion analysis, on these packages is treated as mechanism analysis with standard joints (spherical, revolute). There is a real need to define motion of musculoskeletal system based on the real joint geometry without any "standard joint" assumption. The research work presented here started with this intention of developing formulations and theories to facilitate this. Treating a joint as a standard joint, alters joint tendon, cartilage and muscle loading and results in the loss of muscles which become redundant. The structural level of bone (Rho, KuhnSpearing \& Zioupos, 1998) is composed of the cortical and cancellous bone levels. The most commonly faced bone disease is the osteoporosis, characterized by low bone mass and structural deterioration of bone tissue that can result possibly increasing of bone fractures (Riggs \& Melton, 1995). Usually extra care is taken when treating and reconstructing an osteoporotic bone fracture to avoid any further fracture and implant failure (Schneider, Goldhahn \& Burckhardt, 2005).

\section{LINEAR JOINT MODELLING}

Spring or spring-dashpot elements In this section the transformation of the stiffness matrix of a spring from its local axes frame to the global axes frame will be obtained. The equivalent damping matrix can be obtained by replacing the stiffness coefficients with damping coefficients. In this exercise the three dimensional springs are employed. Thus, three dimensional spring stiffnesses can be described in a local 3D Cartesian frame system. It is considered that the Cartesian frame system coincides with the principal axes of the spring. Therefore,the force deflection equation of the 3D spring element is given as:

\section{$\mathbf{f}=\mathrm{kx}$}

Where $\mathrm{f}$ is a force vector and $\mathrm{k}$ is the stiffness matrix (diagonal with the principal stiffness values) and $x$ is the displacement vector. In general it is convenient to describe the behaviour of a system in the global axes frame (OXYZ). Hence, in order to express the spring stiffness in global axes frame, it needs to be transformed into the global axes frame. It is assumed that the principal axes of the spring and the global axes are all orthogonal, an orthogonal transformation exists between the two frames. Let's assume that transformation matrix $\mathrm{T}$ is used for the transformations from local to global axes frame and $\mathrm{T}^{\mathrm{T}}$ is used for the transformations from global to local axes frame. A vector $\mathrm{x}$ in the local axes frame could be expressed as a vector $X$ in the global axes frame with multiplying it by the transformation matrix $\mathrm{T}$ as:

$$
\mathbf{X}=\mathbf{T x}
$$

And the transformation from global to local is given as:

$$
\mathbf{x}=\mathbf{T}^{\mathrm{T}} \mathbf{X}
$$

Then, the force equation, from the Eqn. 3 can be written in a form as:

$$
\mathbf{f}=\mathrm{kT}^{\mathrm{T}} \mathbf{X}
$$

Therefore, if the Eqn. 4 is pre multiplied by the transformation matrix T, 


\section{$\mathrm{Tf}=\mathrm{TkT}^{\mathrm{T}} \mathbf{X}$}

The force vector $\mathrm{F}$ is described in the global axes frame as:

$$
\mathbf{F}=\mathrm{TkT}^{\mathrm{T}} \mathbf{X}
$$

In short

$$
\mathbf{F}=\mathbf{K X}
$$

Where

$$
\mathbf{K}=\mathbf{T k T}^{\mathrm{T}}
$$

\section{Generalization of the Equation of Linear Momentum}

If the mass/inertia matrix in Newton-Euler formulation is obtained relative to the axes assing through the centre of mass of body $i$, then the subsection of the mass matrix corresponding to linear momentum is a diagonal matrix containing the mass elements, shown in the Eqn. 9

$$
\mathbf{h}_{1}=\mathbf{m v}
$$

Where, $\mathrm{hl}$ is linear momentum, $\mathrm{m}$ is a diagonal matrix and $\mathrm{v}$ is the velocity vector of the centre of mass (COM, casually known as the centre of gravity, COG) of body i in its local axes frame. The usual transformation from local axes frame to global axes frame is also used for transforming linear momentum from the local axes frame to global axes frame as:

$$
\mathbf{H}_{1}=\operatorname{TmT}^{\mathrm{T}} V
$$

It is to be emphasized that mass matrix $\mathrm{m}$ is not changed (all diagonal elements contain the same mass value - mass is not directional). The force acceleration relationship can be obtained from differentiation of the liner momentum. Thus, force acting on body $i$ in the global axes frame can be expressed as:

$$
\mathbf{F}=\dot{\mathbf{H}}_{1}=\frac{\partial \mathbf{H}_{1}}{\partial t}=\mathbf{m a}
$$

For the analysis of rotational motion of a rigid body, the relationship between the angular velocity of the body and the moment of momentum vector is needed. The moment of momentum is expressed as:

$$
\mathbf{h}_{a}=\mathbf{j} \omega
$$

ha is the moment of momentum (angular momentum) vector, $\mathrm{j}$ is the moments of inertia matrix and $\omega$ is the angular velocity vector which are all described in the local axes frame of body i. Here the moments of inertia matrix $\mathrm{j}$ may or may not be a diagonal matrix, however it is always symmetric. Therefore, the moment of momentum vector $h_{a}$ has to be converted in global axes frame, for the final matrix assembly. As presented by the Eqn. 12 for the stiffness matrix, the transformation from local to global axes frame for the inertia matrix follows exactly the same steps. In this case, again, $T$ is the transformation matrix and the angular momentum in the global axes frame is given as:

$$
\mathbf{H}_{a}=\mathrm{Tj}^{\mathrm{T}} \boldsymbol{\Omega}
$$

Similar to the procedure described for the k matrix, inertia matrix in the global axes is given by,

$$
\mathbf{J}=\mathrm{T}_{\mathbf{j}} \mathrm{T}^{\mathrm{T}}
$$


If the vector differentiation is taken for $\mathrm{Ha}$, the moment vector is obtained in the global axes as:

$\mathbf{M}=\dot{\mathbf{H}}_{a}=\frac{\partial \mathrm{H}_{a}}{\partial \mathrm{t}}+\omega \times \mathrm{H}_{a}$

small displacement of di and dj can be described as follows.

$\mathbf{d}_{i}=\mathbf{x}_{i}+\theta_{i} \times \mathbf{r}_{p i}$

$$
\mathbf{d}_{j}=\mathbf{x}_{j}+\theta_{j} \times \mathbf{r}_{p j}
$$

Where, rpi and rpj are coordinates of spring attachment points in their respective axes frames, given as, rpi $=(x p i, y p i, z p i)$ and $r p j=(x p j, y p j, z p j)$. Displacements di and dj are measured in local axes frames, and expressed in matrix form as:

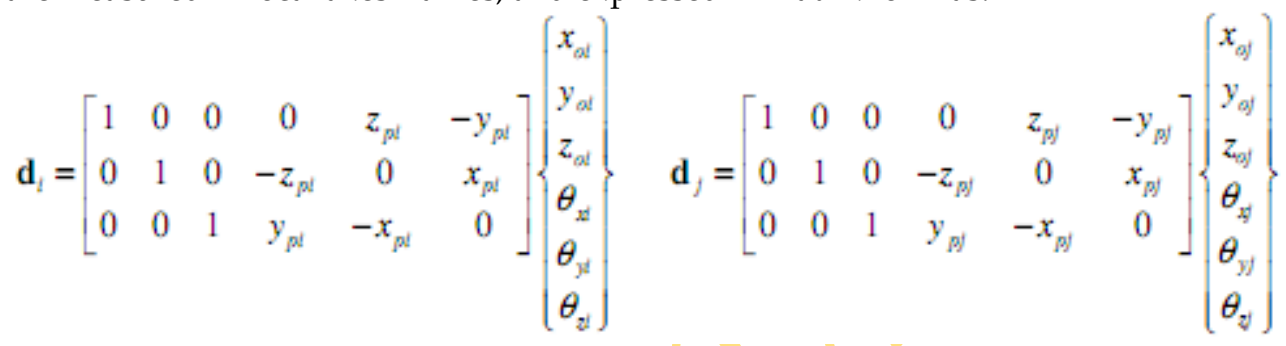

Alternatively, notation di and dj can also be described as:

$$
\begin{array}{r}
\mathbf{d}_{i}=\mathbf{I} \mathbf{x}_{i}+\mathbf{R}_{p i} \theta_{i} \\
\mathbf{d}_{j}=\mathbf{I} \mathbf{x}_{j}+\mathbf{R}_{p j} \theta_{j}
\end{array}
$$

Where

$$
\mathbf{I}=\left[\begin{array}{lll}
1 & 0 & 0 \\
0 & 1 & 0 \\
0 & 0 & 1
\end{array}\right] \quad \mathbf{R}_{p t}=\left[\begin{array}{ccc}
0 & z_{p l} & -y_{p l} \\
-z_{p l} & 0 & x_{p l} \\
y_{p t} & -x_{p l} & 0
\end{array}\right]
$$

The matrix notations of the displacement dj can be obtained by replacing the suffix $\mathrm{i}$ with j. These matrix and vector notations can be combined as Api and ui for displacement di and Apj and uj for displacement dj as:

$$
\begin{aligned}
& \mathbf{A}_{p l}=\left[\begin{array}{cccccc}
1 & 0 & 0 & 0 & z_{p l} & -y_{p l} \\
0 & 1 & 0 & -z_{p l} & 0 & x_{p l} \\
0 & 0 & 1 & y_{p l} & -x_{p l} & 0
\end{array}\right] \quad \mathbf{A}_{p j}=\left[\begin{array}{rrrrrc}
1 & 0 & 0 & 0 & z_{p j} & -y_{p j} \\
0 & 1 & 0 & -z_{p j} & 0 & x_{p j} \\
0 & 0 & 1 & y_{p j} & -x_{p j} & 0
\end{array}\right] \\
& \mathbf{u}_{i}=\left\{\begin{array}{l}
x_{a l} \\
y_{a l} \\
z_{a l} \\
\theta_{x d} \\
\theta_{y j} \\
\theta_{z l}
\end{array}\right\} \quad \mathbf{u}_{j}=\left\{\begin{array}{c}
x_{a j} \\
y_{o j} \\
z_{o j} \\
\theta_{x j} \\
\theta_{y j} \\
\theta_{v j}
\end{array}\right\}
\end{aligned}
$$

Thus di and dj can be expressed in their new forms as: 


$$
\begin{aligned}
& \mathbf{d}_{i}=\mathbf{A}_{p i} \mathbf{u}_{i} \\
& \mathbf{d}_{j}=\mathbf{A}_{p j} \mathbf{u}_{j}
\end{aligned}
$$

Forces and moments acting on body $i$ at position $p$ may be expressed as:

$$
\left\{\begin{array}{l}
F_{p l x} \\
F_{p l y} \\
F_{p l z} \\
T_{p l x} \\
T_{p l y} \\
T_{p l z}
\end{array}\right\} \text { where, }\left\{\begin{array}{l}
T_{p l x} \\
T_{p l y} \\
T_{p l z}
\end{array}\right\}=\mathbf{r}_{p l} \times \mathbf{F}_{p l t} \text { and } \mathbf{F}_{p l t}=\left\{\begin{array}{l}
F_{p l x} \\
F_{p l y} \\
F_{p l z}
\end{array}\right\}
$$

Then, the Eqn. 3.25 may be written in matrix form as:

$$
\left\{\begin{array}{l}
T_{p l x} \\
T_{p l y} \\
T_{p l z}
\end{array}\right\}=\mathbf{r}_{p l} \times \mathbf{F}_{p l}=\left[\begin{array}{ccc}
0 & -z_{p l} & y_{p l} \\
z_{p l} & 0 & -x_{p l} \\
-y_{p i} & x_{p l} & 0
\end{array}\right]\left\{\begin{array}{l}
F_{p l x} \\
F_{p l y} \\
F_{p l z}
\end{array}\right\}
$$

Therefore,

$$
\left\{\begin{array}{c}
F_{p l x} \\
F_{p l y} \\
F_{p l z} \\
T_{p l x} \\
T_{p h y} \\
T_{p l z}
\end{array}\right\}=\left[\begin{array}{ccc}
1 & 0 & 0 \\
0 & 1 & 0 \\
0 & 0 & 1 \\
0 & -z_{p l} & y_{p l} \\
z_{p l} & 0 & -x_{p l} \\
-y_{p l} & x_{p l} & 0
\end{array}\right]\left\{\begin{array}{l}
F_{p l x} \\
F_{p b y} \\
F_{p l z}
\end{array}\right\}
$$

Noting that the matrix in the Eqn18, is the transpose of the matrix introduced in the Eqn. 19 The force equation is written as:

$$
\mathbf{f}_{p i}=\mathbf{A}_{i}^{T} \mathbf{F}_{p i l}
$$

Note that Fpil is a force vector (acting at point p on body i) described in the local axes frame. Normally force is conveniently described in the global axes. This equation can be re-written as:

$$
\mathbf{f}_{p i}=\mathbf{A}_{i}^{T} \mathbf{T}_{i}^{T} \mathbf{F}_{p i(3)}
$$

Now Fpi (3) is in the global axes frame of dimension 3, now pre multiplying the both side of the equation with ' $\mathrm{T}$

$$
\mathbf{F}_{p i}=\mathbf{T}_{i}^{\prime} \mathbf{A}_{i}^{T} \mathbf{T}_{i}^{T} \mathbf{F}_{p i(3)}
$$

The left hand side of the equation has dimension 6 . Where;

$$
\mathbf{T}_{i}=\left[\begin{array}{cc}
\mathbf{T}_{i} & 0 \\
0 & \mathbf{T}_{i}
\end{array}\right]
$$

Now, forces acting on body $i$ is written as (for this, point deflections are described in the global axes):

$$
\begin{aligned}
& \mathbf{F}_{p i(3)}=\mathbf{k}_{p}\left(\mathbf{T}_{j} \mathbf{d}_{p j}-\mathbf{T}_{i} \mathbf{d}_{p i}\right) \\
& \mathbf{F}_{p i(3)}=\mathbf{k}_{p}\left(\mathbf{T}_{j} \mathbf{A}_{p j} \mathbf{u}_{j}-\mathbf{T}_{i} \mathbf{A}_{p i} \mathbf{u}_{i}\right)
\end{aligned}
$$


$\mathrm{u}$ Vectors are still in the local frame and it needs to be replaced by $\mathrm{U}$.

$\mathbf{F}_{p i(3)}=\mathbf{k}_{p}\left(\mathbf{T}_{j} \mathbf{A}_{p j} \mathbf{T}_{j}^{T} \mathbf{U}_{j}-\mathbf{T}_{i} \mathbf{A}_{p i} \mathbf{T}_{i}^{T} \mathbf{U}_{i}\right)$

$\mathrm{kp}$ is described in the global axes frame, Fpi is a force vector in the global axes frame, the following steps are self explanatory.

$$
\begin{aligned}
& \mathbf{M}_{i} \ddot{\mathbf{u}}_{i}=\sum_{\rho} \mathbf{f}_{p i} \\
& \mathbf{M}_{i} \mathbf{T}_{i}^{i T} \ddot{\mathbf{U}}=\sum_{p} \mathbf{f}_{p i} \\
& \mathbf{T}_{i}^{\prime} \mathbf{M}_{i} \mathbf{T}_{i}^{\tau} \ddot{\mathbf{U}}=\mathbf{T}_{i}^{i} \sum_{p} \mathbf{f}_{\rho i} \\
& \mathbf{T}_{i}^{\prime} \mathbf{M}_{i} \mathbf{T}_{i}^{\tau} \ddot{\mathbf{U}}=\sum_{\rho} \mathbf{F}_{p i}
\end{aligned}
$$

Substituting forces from the Eqn. 22 into the Eqn. 23 and into the Eqn. 24 for suffix $i$ and $\mathrm{j}$, the final equation of motions may be written as:

$$
\begin{aligned}
& \mathbf{T}_{i}^{\prime} \mathbf{M}_{i} \mathbf{T}_{i}^{\tau \tau} \ddot{\mathbf{U}}_{i}+\sum_{p}\left(\mathbf{T}_{i}^{i} \mathbf{A}_{i}^{\tau} \mathbf{T}_{i}^{\tau} \mathbf{k}_{\rho} \mathbf{T}_{i} \mathbf{A}_{\rho i} \mathbf{T}_{i}^{\tau}\right) \mathbf{U}_{i}-\sum_{\rho}\left(\mathbf{T}_{i}^{i} \mathbf{A}_{i}^{\tau} \mathbf{T}_{i}^{\tau} \mathbf{k}_{\rho} \mathbf{T}_{j} \mathbf{A}_{\rho j} \mathbf{T}_{j}^{\tau}\right) \mathbf{U}_{j}=0 \\
& \mathbf{T}_{j}^{\prime} \mathbf{M}_{j} \mathbf{T}_{j}^{\tau} \ddot{\mathbf{U}}_{j}-\sum_{p}\left(\mathbf{T}_{j}^{i} \mathbf{A}_{j}^{\tau} \mathbf{T}_{j}^{\tau} \mathbf{k}_{\rho} \mathbf{T}_{i} \mathbf{A}_{\rho i} \mathbf{T}_{i}^{\tau}\right) \mathbf{U}_{i}+\sum_{\rho}\left(\mathbf{T}_{j}^{\prime} \mathbf{A}_{j}^{\tau} \mathbf{T}_{j}^{\tau} \mathbf{k}_{\rho} \mathbf{T}_{j} \mathbf{A}_{\rho j} \mathbf{T}_{j}^{\tau}\right) \mathbf{U}_{j}=0
\end{aligned}
$$

the Eqn. 24 and Eqn. 25 can be written in the following formats as:

$$
\begin{aligned}
& {\left[\begin{array}{cc}
\mathbf{m}_{i} & 0 \\
0 & \mathbf{J}_{i}
\end{array}\right]\left\{\begin{array}{l}
\ddot{\mathbf{x}}_{i} \\
\ddot{\boldsymbol{\theta}}_{i}
\end{array}\right\}-\left[\begin{array}{cc}
\mathbf{k}_{p} & \mathbf{k}_{p} \mathbf{R}_{p i} \\
\mathbf{R}_{p i}^{\mathrm{T}} \mathbf{k}_{p} & \mathbf{R}_{p i}^{\mathrm{T}} \mathbf{k}_{p} \mathbf{R}_{p i}
\end{array}\right]\left\{\begin{array}{l}
\mathbf{x}_{i} \\
\boldsymbol{\theta}_{i}
\end{array}\right\}+\left[\begin{array}{cc}
\mathbf{k}_{p} & \mathbf{k}_{p} \mathbf{R}_{p j} \\
\mathbf{R}_{p i}^{\mathrm{T}} \mathbf{k}_{p} & \mathbf{R}_{p i}^{\mathrm{T}} \mathbf{k}_{p} \mathbf{R}_{p j}
\end{array}\right]\left\{\begin{array}{l}
\mathbf{x}_{j} \\
\boldsymbol{\theta}_{j}
\end{array}\right\}=\left\{\begin{array}{c}
\mathbf{F}_{i} \\
\mathbf{M}_{i}
\end{array}\right\}} \\
& {\left[\begin{array}{cc}
\mathbf{m}_{j} & 0 \\
0 & \mathbf{J}_{j}
\end{array}\right]\left\{\begin{array}{l}
\ddot{\mathbf{x}}_{j} \\
\ddot{\boldsymbol{\theta}}_{j}
\end{array}\right\}+\left[\begin{array}{cc}
\mathbf{k}_{p} & \mathbf{k}_{p} \mathbf{R}_{p i} \\
\mathbf{R}_{p i n}^{\mathrm{T}} \mathbf{k}_{p} & \mathbf{R}_{p i}^{\mathrm{T}} \mathbf{k}_{p} \mathbf{R}_{p i}
\end{array}\right]\left\{\begin{array}{l}
\mathbf{x}_{i} \\
\boldsymbol{\theta}_{i}
\end{array}\right\}-\left[\begin{array}{cc}
\mathbf{k}_{p} & \mathbf{k}_{p} \mathbf{R}_{p j} \\
\mathbf{R}_{p j}^{\mathrm{T}} \mathbf{k}_{p} & \mathbf{R}_{p_{j}}^{\mathrm{T}} \mathbf{k}_{p} \mathbf{R}_{p_{j}}
\end{array}\right]\left\{\begin{array}{l}
\mathbf{x}_{j} \\
\boldsymbol{\theta}_{j}
\end{array}\right\}=\left\{\begin{array}{c}
\mathbf{F}_{j} \\
\mathbf{M}_{j}
\end{array}\right\}}
\end{aligned}
$$

Therefore, through eliminating the motion of the body j, the Eqn. 25 is expressed for body i as:

$$
\mathbf{T}_{i}^{i} \mathbf{M}_{i} \mathbf{T}_{i}^{T} \mathbf{U}_{i}+\sum_{p}\left(\mathbf{T}_{i}^{\prime} \mathbf{A}_{i}^{T} \mathbf{T}_{i}^{T} \mathbf{k}_{p} \mathbf{T}_{i} \mathbf{A}_{p i} \mathbf{T}_{i}^{\prime T}\right) \mathbf{U}_{i}=0
$$

The question one that needs to answer is how to use the matrix such a way that it gives practical information with respect to joint laxity measurement. Firstly the stiffness needs to be measured to axes relative to the joint itself and translational stiffness (trying to pull the joint apart) has to be separated from the rotational ones. And the rotational stiffnesses have to be identified relative to the instantaneous axis of rotation. In order to achieve this, the translational stiffness should not be coupled with the rotational stiffness. Although the Eigen value analysis of the stiffness matrix achieves this precisely, it is not always easy to give a physical interpretation to the Eigen values or vectors. However there is a more direct way of achieving this. This concept, to be called as the centre of stiffness (similar to centre of mass) and it has the property that stiffness formulation with respect to this centre will have translational stiffness completely uncoupled from the rotational stiffness. The calculation of the centre of the 
rotation can be defined as the centre, relative to which any rotation will not generate a resultant force. In vectorial terms this can be expressed as:

$$
\boldsymbol{k}_{i}\left(\boldsymbol{\theta} \times\left(\mathbf{r}_{i}-\boldsymbol{\rho}_{i}\right)\right)=0
$$

Where ki is the stiffness matrix of the ith tissue in the global axis (this is expressed in the global axis for convenience before assembling the formulation for the sake of simplicity). $\theta$ is the arbitrary rotation vector and $\mathrm{ri}$ is the position of the ith tissue attachment on body $i$ (remembering that body $j$ is fixed in space) all measured relative to the local body axes (with axes parallel to the global axes, which does not need any transformation). $\rho$ is the position vector of the centre of stiffness. The equation now can be expressed, for all the stiffness elements as:

$$
\sum_{\text {difissues }} k_{i}\left(\boldsymbol{\theta} \times \mathbf{r}_{i}\right)-k_{i}(\boldsymbol{\theta} \times \boldsymbol{\rho})=0
$$

Now replacing the vector equation with its matrix equivalent is given as:

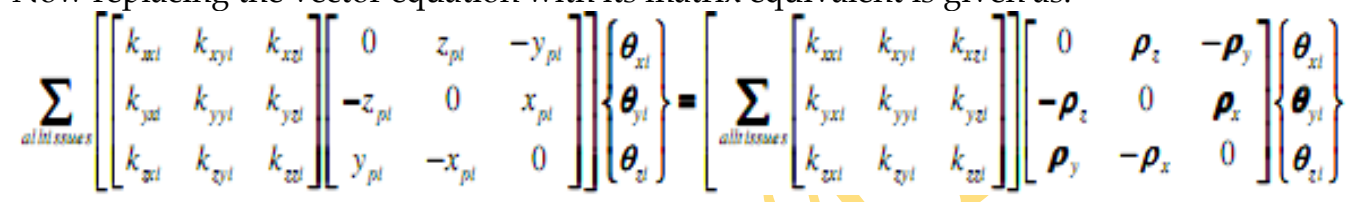

$$
\begin{aligned}
& {\left[\sum\left(-z_{p i} k_{s j i}+y_{p i} k_{z z i}\right) \sum\left(z_{p i} k_{x z i}-x_{p i} k_{z z i}\right) \sum\left(-y_{p i} k_{x z i}+x_{p i} k_{x j i}\right)\right.}
\end{aligned}
$$

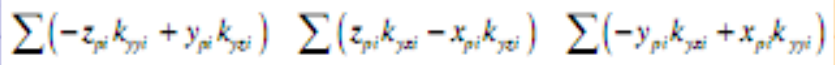

$$
\begin{aligned}
& \sum\left(-z_{p i} k_{z i j}+y_{p i} k_{z i}\right) \quad \sum\left(z_{p i} k_{z z i}-x_{p i} k_{z i}\right) \quad \sum\left(-y_{p i} k_{z z i}+x_{p i} k_{z j i}\right)
\end{aligned}
$$

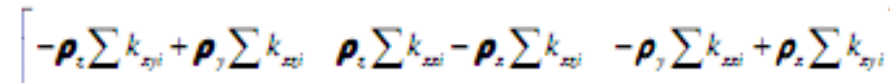

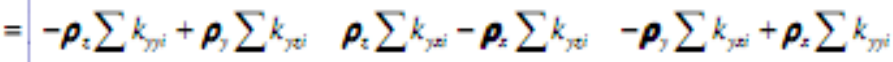

$$
\begin{aligned}
& {\left[\begin{array}{lll}
-\boldsymbol{\rho}_{z} \sum k_{z i j}+\boldsymbol{\rho}_{7} \sum k_{z z} & \boldsymbol{\rho}_{z} \sum k_{z z i}-\boldsymbol{\rho}_{z} \sum k_{z t i} & -\boldsymbol{\rho}_{7} \sum k_{z z i}+\boldsymbol{\rho}_{z} \sum k_{z j i}
\end{array}\right]}
\end{aligned}
$$

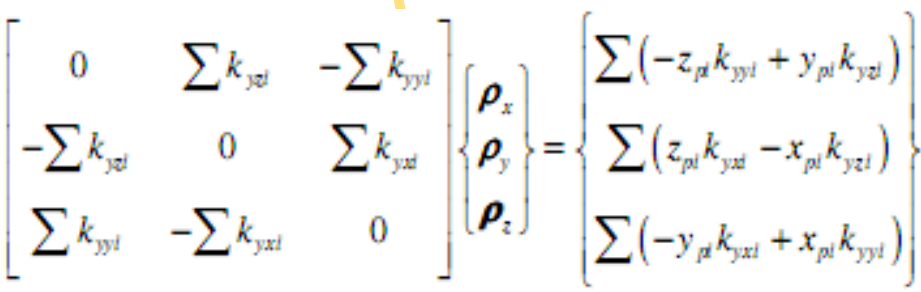

The following equations are found to satisfy all the conditions for a valid solution.

$$
\begin{aligned}
& -\rho_{z} \sum k_{y y i}+\rho_{y} \sum k_{y z i}=\sum\left(-z_{p i} k_{y y i}+y_{p i} k_{y z i}\right) \\
& -\rho_{z} \sum k_{z y i}+\rho_{y} \sum k_{z z i}=\sum\left(-z_{p i} k_{z y i}+y_{p i} k_{z z i}\right)
\end{aligned}
$$




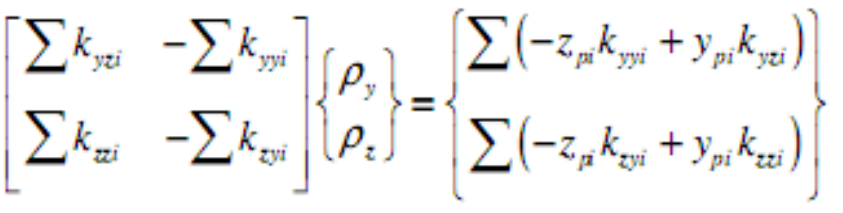

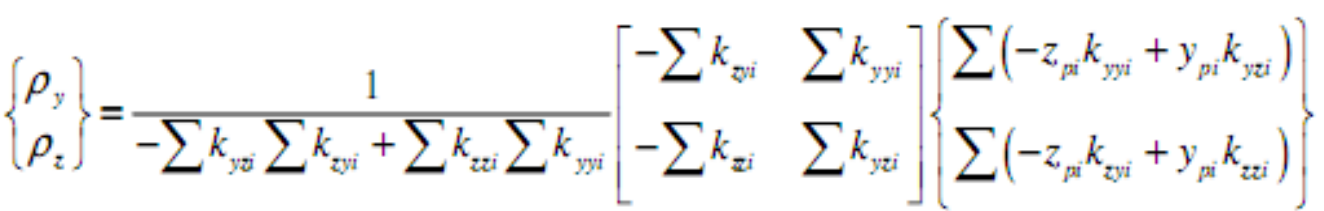

The formulation above ensures that there is always a solution provided, kzz, kyy exist as they both appear in the denominator and the nominator of the expression. And final from is given by:

$$
\begin{aligned}
& -\rho_{y} \sum k_{y x i}+\rho_{x} \sum k_{y y i}=\sum\left(-y_{p i} k_{y x i}+x_{p i} k_{y y i}\right) \\
& \rho_{x}=\frac{1}{\sum k_{y y i}}\left(\rho_{y} \sum k_{y x i}+\sum\left(-y_{p i} k_{y x i}+x_{p i} k_{y y i}\right)\right)
\end{aligned}
$$

Where, again the nominator and denominator contain diagonal stiffness elements which ensure that there is always a feasible solution. To illustrate what is happening, one can consider a single spring with its principal axes coinciding with the global axes. This way one can see what may happen in a situation where matrix is reduced to following form as:

$$
\left[\begin{array}{cccccc}
k_{p x} & 0 & 0 & 0 & k_{p x} z_{p} & -k_{p x} y_{p} \\
0 & k_{p y} & 0 & -k_{p y} z_{p} & 0 & k_{p y} x_{p} \\
0 & 0 & k_{p z} & k_{p z} y_{p} & -k_{p r} x_{p} & 0 \\
0 & -k_{p p} z_{p} & k_{p z} y_{p} & k_{p p} y_{p}^{2}+k_{p y} z_{p}^{2} & -k_{p p} x_{p} y_{p} & -k_{p y} x_{p} z_{p} \\
k_{p x} z_{p p} & 0 & -k_{p p} x_{p} & -k_{p p} x_{p} y_{p} & k_{p p} x_{p}^{2}+k_{p x} z_{p}^{2} & -k_{p x} y_{p} z_{p} \\
-k_{p x} y_{p} & k_{p p} x_{p} & 0 & -k_{p p} x_{p} z_{p} & -k_{p x} y_{p} z_{p p} & k_{p p} x_{p}^{2}+k_{p x} y_{p}^{2}
\end{array}\right]
$$

\section{Applications, Results and Validation}

In order to create the proposed 6DOF joint models, surface geometries of bones (segments) are needed. The morphologic study of cadaveric specimens has been performed and bones have been digitized using FARO Platinum Arm by previous researchers in the Brunel Orthopaedic Research and Learning Centre. The output data from the digitization is obtained as point clouds with imperfect surface conditions in the IGES file format. Thus, in order to generate meshed surface data, the Geomagic Studio 9 has been utilized (GEOMAGIC). The procedure starts with importing the iges files into the Geomagic Studio The point cloud of raw data is usually obtained in different conditions and each bone can possess more than thirty thousand points on its surface. According to the results obtained from Lifemod software, head and neck movements are depicted as below. The analysis has been performed for frontal impact. The head mass is $11.1743833572 \mathrm{lb}(5.068615 \mathrm{~kg})$ and neck mass is $2.9014822662 \mathrm{lb}(1.316 \mathrm{~kg})$. Joints are created based on the Hybrid III crash Dummy based strength values with the Hybrid 
III scale factor 1 from the Lifemod software. As mentioned earlier these factors are used in order to provide the stiffness values for each rotational direction as in sagittal, transverse and frontal planes. Ligaments are interspinous ligaments, flaval ligaments, facet joint capsule ligaments and longitudional ligaments have been created from head through cervical vertebrae (C1-C7) to thoracic vertebra (T1). Basically ligaments are modelled as viscoelastic material with parallel spring and dashpot (Kelvin-Voigt model) elements with $100 \mathrm{lbf} /$ in stiffness and $20 \mathrm{lbf} /$ in/s damping values (these are $20 \mathrm{kN} / \mathrm{m} 4 \mathrm{kN} / \mathrm{m} / \mathrm{s}$ ). Muscles are semispinalis cervicis, trapezius, longus colli, semispinalis capitis and sternocleidomastoid muscles and modelled based on the Hill type muscle model. All required passive material properties and active ontraction properties have been adapted from the Lifemod library. The dynamic analysis is based on the given translational acceleration to upper torso. In order to impose the acceleration the upper torso is represented as a translational joint where the translational velocity is created based on this constrained joint movement. The total simulation time is $0.5 \mathrm{sec}$, where the acceleration is applied around $0.15-0.17$ seconds and off at 0.2 seconds. The profile of the acceleration is a "half sine" shock which is convenient as it is one of the available options in the MJM force library. After completing the dynamic analysis variety of results are depicted as follows.

BoOr seOMent wonON

in_estosn

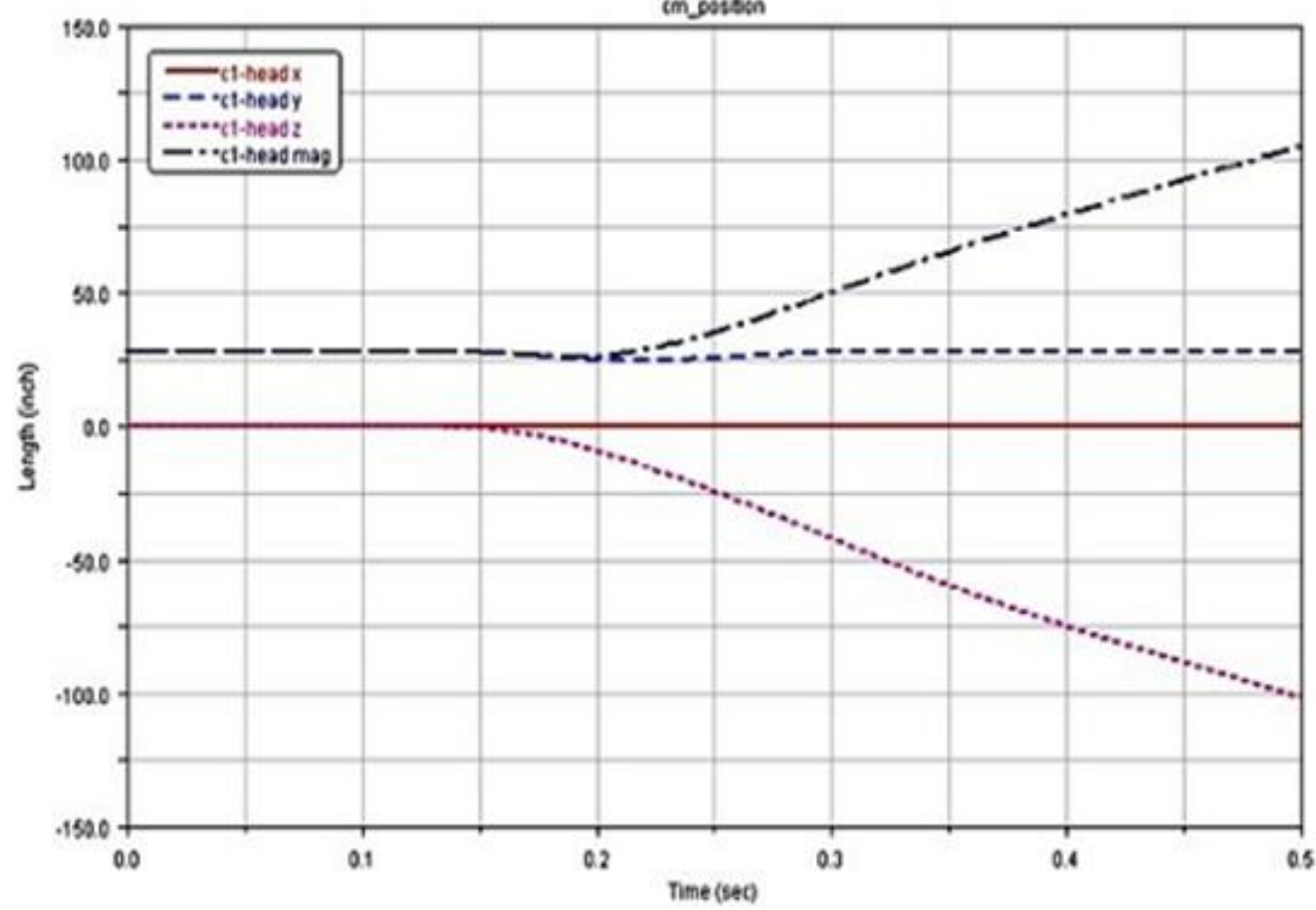

Figure 1 Centre of mass position of head versus time response to frontal impact applied to the upper torso

Results shown in the Figure 1. are very similar to the result obtained from the MJM simulations. The only difference is the starting time for the shock. In MJM the shock starts immediately whereas in the Lifemod software there is approximately a delay of 0.15s. As these results are from the demonstration case study provided by the Lifemod 
software, it was not possible to make changes to study alternative cases. Ignoring the actual response initiation, the results are almost identical. Velocity and angle plots of the head are similarly in good agreement as shown in Figure 2. and Figure 3. respectively. The substantial difference between these results and MJM is the lateral motion. In the Lifemod software, there is a complete symmetry, whether this is realistic in the real life, is debateable, the results are two dimensional, there are movements in the $\mathrm{z}$ and the $\mathrm{x}$ directions but none in the $\mathrm{y}$ direction. And the angle rotation is only in $\mathrm{y}$ direction (beta in MJM terms) due to fixed joint between the head and C1.

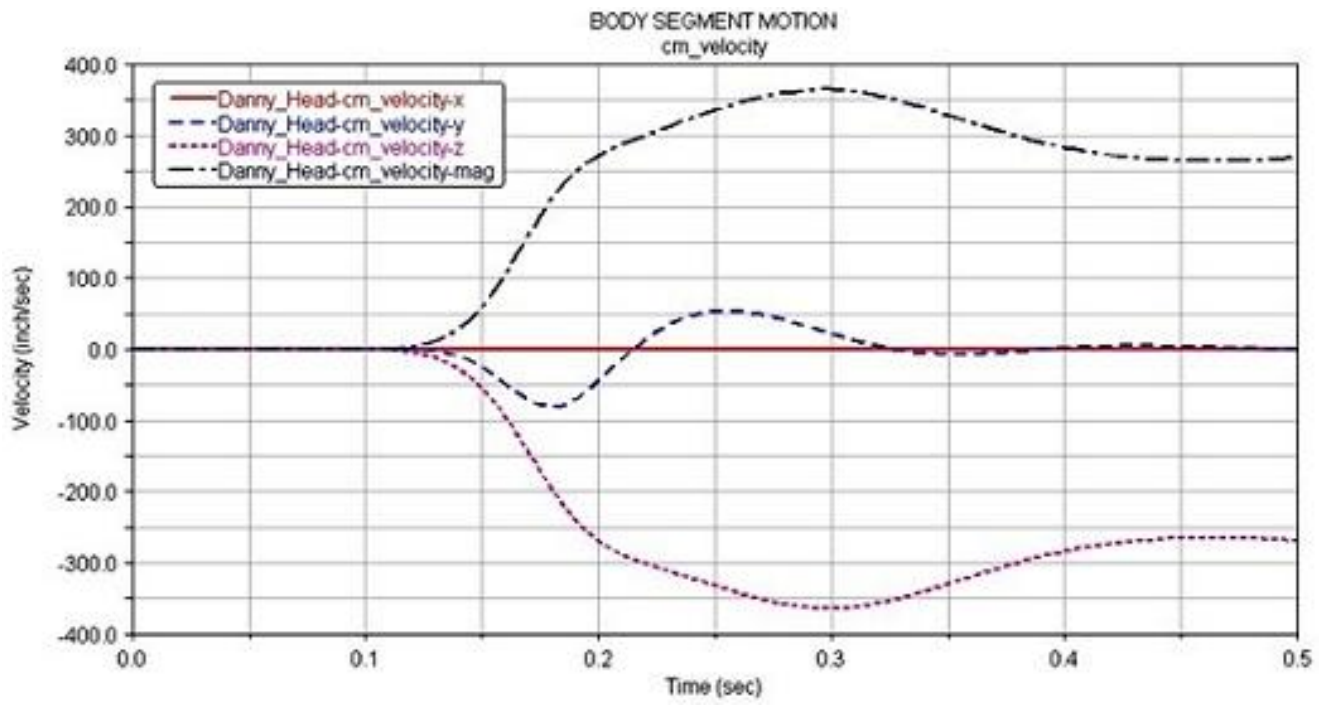

Figure 2 Centre of mass velocity of head versus time response to frontal impact applied to the upper torso

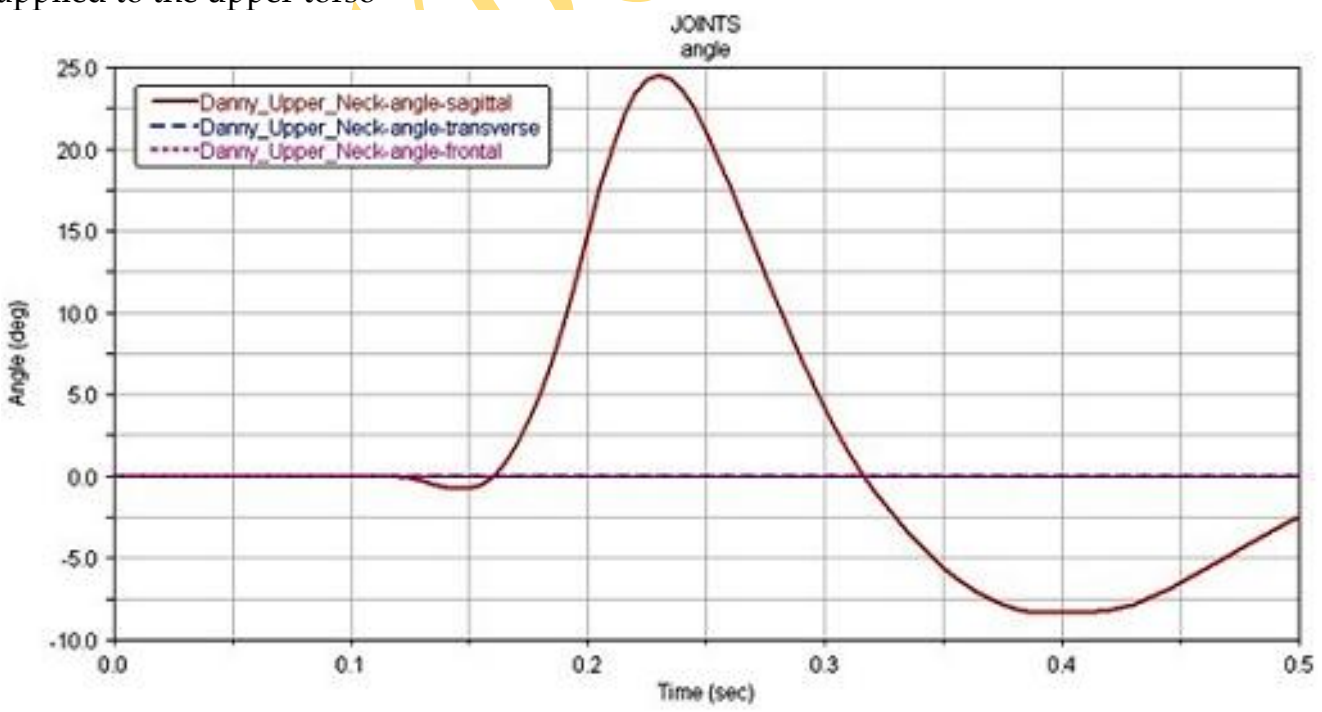

Figure 3 Angle of upper neck (joint between Head-C1) versus time response to frontal impact applied to the upper torso which shows one dimensional rotation in sagittal plane (horizontal direction) 


\section{Conclusions}

The work reported in this thesis, encapsulates the theories and algorithms developed to drive the core analysis modules of the software which was developed to model a musculoskeletal structure and particularly the diarthrodial and amphiarthroses joints. What makes the proposed modeller different than currently available modellers is that the joint kinematics is based on the local joint surfaces and contact geometry. There are many modellers capable of modelling gross human body motion. Nevertheless, none of the available modellers offers complete elements of joint modelling, it appears that joint modelling is an extension of their core analysis capability which is the musculoskeletal motion dynamics. It is felt that an analysis framework focused on human joints would offer a significant benefit and potential to be used in surgical environments. The local mobility of joints has a significant influence in human motion analysis, and in understanding of joint loading or contact forces. Thus, an accurate analysis of joint motion is very important in medical applications such as implant evaluation or surgery assistance and assisting medical devices and instrumentations.

\section{REFERENCES}

Abdel Rahman E., Hefzy M., (1998) “Three Dimensional Dynamic Behaviour of the Human Knee Joint under Impact Loading," Medical Engineering \& Physics 20(4), pp.276-290.

Abdel Rahman E., Hefzy M., (1993) “A Two Dimensional Dynamic Anatomical Model of the Human Knee Joint," Journal of Biomechanics 115, pp. 357-364.

Abdel-Malek K., Yang J., Marler T., Beck S., Mathai A., Zhou X., Patrick A., Arora J., (2006) "Towards a New Generation Virtual Humans: Santos," International Journal of Human Factors Modeling and Simulation 1, pp. 2-39.

Ackland D., Pak P., Richardson M., Pandy M., (2008) "Moment Arms of the Muscles Crossing the Anatomical Shoulder," Journal of Anatomy 213, pp. 383-390.

Adams C., Baldwin M., Laz P., Rullkoetter P., Langenderfer J., (2007) “Effects of Rotator Cuff Tears on Muscle Moment Arms: A Computational Study," Journal of Biomechanics 40, pp. 3373-3380.

Ahmad C., Park M., ElAttrache N., (2004) “Elbow Medial Ulnar Collateral Ligament Insufficiency Alters Posteromedial Olecranon Contact," The American Journal of Sports Medicine 32(7), pp. 1607-1612.

Alrashidi M., Yildiz I., Alrashdan K., Esat I., (2009) “Evaluating Elbow Joint Kinematics with the Stewart Platform Mechanism," Eighth International Conference on Modelling in Medicine and Biology.

Amevo B., Aprill C., Bogduk N., (1992) “Abnormal Instantaneous Axes of Rotation in Patients with Neck Pain," Spine 17(7), pp. 748-756.

An K., (2005) "Kinematics and Constraint of Total Elbow Arthroplasty," Journal of Shoulder and Elbow Surgery 14, pp. 168S-173

\section{Why Open Access ???}

"In the traditional publishing model, readers have limited access to scientific papers; authors do not have copyright for their own papers, and cannot post their papers on their own websites, which presents a significant barrier to the sharing of knowledge, as well as being unfair to authors. Open access can overcome the drawbacks of the traditional publishing model and help scholars build on the findings of their colleagues without restriction" 